\title{
A New Approach for Simple and Rapid Shape Measurement of Objects with Surface Discontinuities
}

\author{
Sai Siva Gorthi* and Kameswara Rao Lolla \\ Department of Instrumentation, Indian Institute of Science, Bangalore 560012, INDIA
}

\begin{abstract}
A new approach for unwrapping phase maps, obtained during the measurement of 3-D surfaces using sinusoidal structured light projection technique, is proposed. "Takeda's method" is used to obtain the wrapped phase map. Proposed method of unwrapping makes use of an additional image of the object captured under the illumination of a specifically designed color-coded pattern. The new approach demonstrates, for the first time, a method of producing reliable unwrapping of objects even with surface discontinuities from a single-phase map. It is shown to be significantly faster and reliable than temporal phase unwrapping procedure that uses a complete exponential sequence. For example, if a measurement with the accuracy obtained by interrogating the object with $\mathrm{S}$ fringes in the projected pattern is carried out with both the methods, new method requires only 2 frames as compared to $\left(\log _{2} \mathrm{~S}+1\right)$ frames required by the later method.
\end{abstract}

Keywords: Shape measurement, FFT analysis, fringe pattern, phase unwrapping, color-coding, surface discontinuities.

\section{INTRODUCTION}

The measurement of surface shape by use of projected structured light patterns is a well-developed technique. In this sinusoidal fringe pattern projection method is most widely used owing to its simplicity, accuracy and high spatial resolution measurement capabilities. It allows the measurement of topographical variations of the object surface based on the retrieval of phase information encoded in the fringe pattern. Fourier transform ${ }^{1}$, phase stepping methods ${ }^{2}$ are the best popular fringe analysis techniques used to calculate the underlying phase distribution of the deformed fringe pattern. A major obstacle that frustrates the use of sinusoidal structured light projection technique is that the recovered phase using afore mentioned fringe analysis techniques is mathematically limited to the interval $[-\pi, \pi]$ corresponding to the principle value of arc tan function. In general the true phase may range over an interval greater than $2 \pi$ in which case the recovered phase contains artificial discontinuities. Unwrapping these discontinuities is a matter of adding an appropriate integer multiple of $2 \pi$ to each pixel element of the wrapped phase map. Normal phase unwrapping is carried out by comparing the phase at neighboring pixels and adding or subtracting $2 \pi$ to bring the relative phase between the two pixels into the range of $-\pi$ to $\pi$. This causes problems when the technique is applied to real objects in engineering or medical measurements, because such objects contain edges or discontinuities that cause the true phase jump between any two pixels lay out side this range ${ }^{3}$. It can then become impossible to unwrap correctly across such real discontinuities and large (multiples of $2 \pi$ ) phase errors can propagate across the image.

To overcome this problem several phase unwrapping strategies were developed. Temporal phase unwrapping4, Reduced temporal phase unwrapping ${ }^{5,6}$, Spatio-temporal phase unwrapping ${ }^{7,8}$ and Frequency multiplexing ${ }^{9,10}$ procedures are the popular ones. However, to perform phase unwrapping, all these methods mandataroly require multiple phase maps generated by varying the spatial frequency of projected fringe pattern either linearly or exponentially. Further, the degree of reliability varies from method to method.

A different class of structured light projection techniques relies upon color-coded projection. In contrast to sinusoidal structured light projection, color-coded projection technique does not involve any phase unwrapping process. The color-coded illumination of the object supplies an additional degree of freedom that is necessary for measuring a surface profile ${ }^{11}$.

* E-mail: saisiva@ isu.iisc.ernet.in; Phone 080-22932741; Fax: +91-080-23600135. 
Weiyi Liu et al. ${ }^{12}$ proposed a color-coded projection grating method for shape measurement with a single exposure. Eight different colors were used to code the projection grating. Limitations of such methods are the spatial resolution of the measurement is far less than the fringe projection method, measurement is discontinuous in one of the directions and fineness of measurement in that direction is approximately equal to the width/pitch of the bands used in projection.

There have been some reports on using color as a means to boost the profiling speed while keeping the high resolution of fringe projection technique ${ }^{13,14}$. In this context multichannel approach (that uses interlaced RGB sinusoidal pattern) is effective in reducing the number of frames required by thrice compared to conventional procedures, but still multiple phase maps are required for reliable unwrapping of objects with surface discontinuities ${ }^{15,16}$. Moreover, previous experimental results showed that the measurement accuracy of this technique was lower than that of the traditional Gray scale technique due largely to color coupling and imbalance problems.

The main interest in this work is to solve the problem of discontinuities, especially those caused by the object with height steps. The new approach proposed in this paper is based on the use of an image of object captured under the illumination of a specifically designed color-coded pattern along with a single phase map obtained through Takeda's method.

\section{METHOD}

The first step of profiling objects in the proposed method involves the generation of wrapped phase map using "Takeda's method". The fundamental concepts of Fourier transform profilometry are explained briefly in sec-2.1. The optical setup for Fourier transform profilometry system with crossed optical axis geometry is as shown in Fig.1.

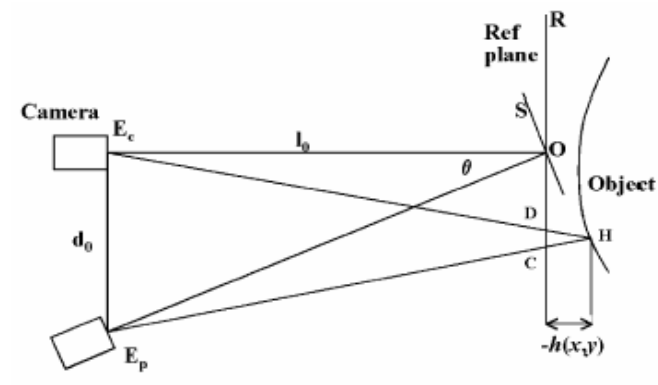

Projector

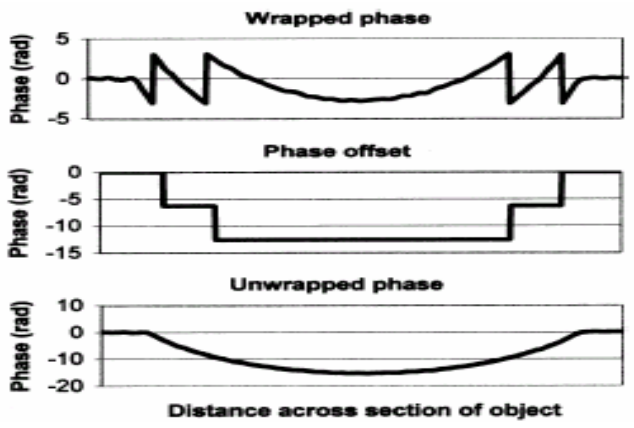

Figure-1 Optical arrangement

Figure-2 Conventional spatial phase unwrapping

\subsection{Fourier Transform Profilometry (Takeda's Method)}

The intensity profile of the recorded image when fringe pattern is projected on to the object is given by

$$
\mathrm{g}(\mathrm{x}, \mathrm{y})=\mathrm{a}(\mathrm{x}, \mathrm{y})+\mathrm{b}(\mathrm{x}, \mathrm{y}) \cos \left(2 \pi \mathrm{f}_{0} \mathrm{x}+\varphi(\mathrm{x}, \mathrm{y})\right)
$$

where $\varphi(x, y)$ contains the desired information and $a(x, y), b(x, y)$ represent unwanted irradiance variations arising from the non-uniform light reflection by a test object. This equation can be rewritten as

$$
\begin{gathered}
g(x, y)=a(x, y)+c(x, y) \exp \left(j 2 \pi f_{0} x\right)+c *(x, y) \exp \left(-j 2 \pi f_{0} x\right) \\
\text { where } c(x, y)=1 / 2 b(x, y) \exp (j \varphi(x, y))
\end{gathered}
$$


* denote complex conjugate. Next Eq. (2) is Fourier transformed with respect to $\mathrm{x}$ by the use of a fast Fourier transform algorithm, which gives

$$
G(f, y)=A(x, y)+C\left(f-f_{0}, y\right)+C *\left(f-f_{0}, y\right)
$$

where capital letters denote the Fourier spectrum and $\mathrm{f}$ is the spatial frequency in the $\mathrm{x}$ direction. Since the spatial variations of $\mathrm{a}(\mathrm{x}, \mathrm{y}), \mathrm{b}(\mathrm{x}, \mathrm{y})$ and $\varphi(\mathrm{x}, \mathrm{y})$ are slow compared with the spatial frequency $\mathrm{f}_{0}$, the Fourier spectra in above equation are separated by the carrier frequency $\mathrm{f}_{0}$. One can make use of either of the two spectra centered around the carrier, say $C\left(f-f_{0}, y\right)$ and translates it by $f_{0}$ on the frequency axis towards the origin to obtain $C(f, y)$. The unwanted background variations a $(x, y)$ has been filtered out in this stage. Again using inverse FFT, inverse Fourier transform of C (f, y) w.r.to $\mathrm{f}$ is computed to obtain $\mathrm{c}(\mathrm{x}, \mathrm{y})$. The phase part of $\mathrm{c}(\mathrm{x}, \mathrm{y})$ can be separated from the rest by calculating the imaginary part of the complex logarithm:

$$
\varphi(x, y)=\operatorname{Im}\{\log [c(x, y)]\}
$$

The phase map for the whole image is formed by repeating this process for each horizontal line in the image. However the phase thus obtained is indeterminate to a factor of $2 \pi$ (wrapped phase). The phase unwrapping algorithm can correct these discontinuities.

\subsection{Phase Unwrapping}

The object phase $\varphi(x, y)$ calculated according to Eq.(5) is wrapped in the range $-\pi$ to $\pi$. The true phase of the object is

$$
\begin{aligned}
\varphi_{\text {un }}(\mathrm{x}, \mathrm{y}) & =\varphi(\mathrm{x}, \mathrm{y})+2 \mathrm{n}(\mathrm{x}, \mathrm{y}) \pi \\
& =\varphi(\mathrm{x}, \mathrm{y})+\varphi_{\text {off }}(\mathrm{x}, \mathrm{y})
\end{aligned}
$$

where $\varphi_{\mathrm{un}}(\mathrm{x}, \mathrm{y}), \varphi(\mathrm{x}, \mathrm{y})$ and $\varphi_{\text {off }}(\mathrm{x}, \mathrm{y})$ are unwrapped phase, wrapped phase and off-set phase respectively. $\mathrm{n}(\mathrm{x}, \mathrm{y})$ is an integer. Unwrapping is only a process of determining $\mathrm{n}(\mathrm{x}, \mathrm{y})$. A conventional spatial phase unwrapping algorithm (CSPU) search for locations of phase jumps in the wrapped phase distribution and adds/subtracts $2 \pi$ to bring the relative phase between two neighboring pixels into the range of $-\pi$ to $\pi$. Thus irrespective of actual value of $n(x, y)$ to be evaluated, they always assign \pm 1 thereby fails to reliably unwrap phase maps in profiling objects with surface discontinuities explained in Ref. 1 procedure:

(where $\mathrm{n}(\mathrm{x}, \mathrm{y})$ can be greater than one). Fig.2 illustrates the conventional phase unwrapping as In order to determine $\mathrm{n}(\mathrm{x}, \mathrm{y})$ (equivalently $\left.\phi_{\text {off }}(\mathrm{x}, \mathrm{y})\right)$ we are introducing the following

\subsection{New Unwrapping Procedure}

In this new approach, an additional image of the object captured under illumination of a color-coded pattern is used for calculating $\mathrm{n}(\mathrm{x}, \mathrm{y})$. A color-coded pattern is generated using MATLAB in a computer and projected with the help of a LCD projector. Color-coded pattern generated comprises of: an array of rectangular shaped bands, with each band identified uniquely by its color and arranged in a specific sequence as shown in Fig.3. This pattern is projected onto the reference plane and its image $\left(\mathrm{C}_{\mathrm{r}}(\mathrm{i}, \mathrm{j})\right)$ is recorded. A non-planar real object distorts the projected structured pattern in accordance with its topographical variations $\left(\mathrm{C}_{\mathrm{o}}(\mathrm{i}, \mathrm{j})\right)$. Now, we know a priori the color expected to be returned by every point from an ideally planar object surface. Deviations in the detected color at any point on the non-planar object surface essentially correspond to local height deviations.

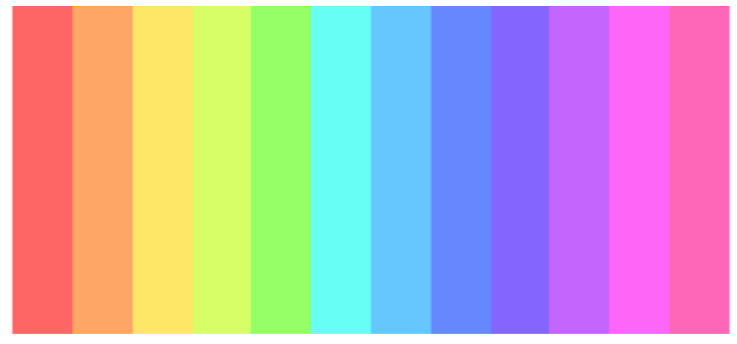


Figure-3 Generated color-coded pattern

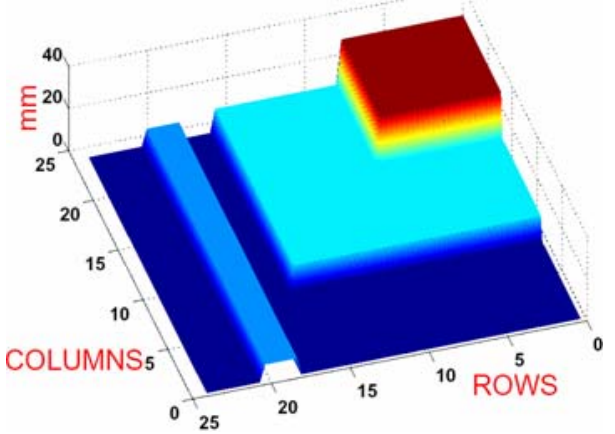

Figure-4 Object with Step discontinuities

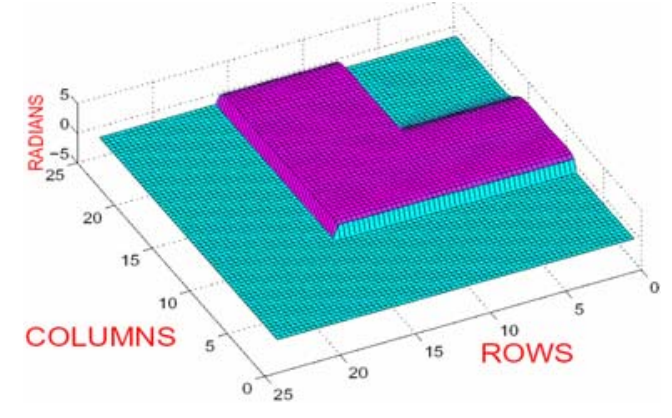

Figure-6 wrapped phase distribution
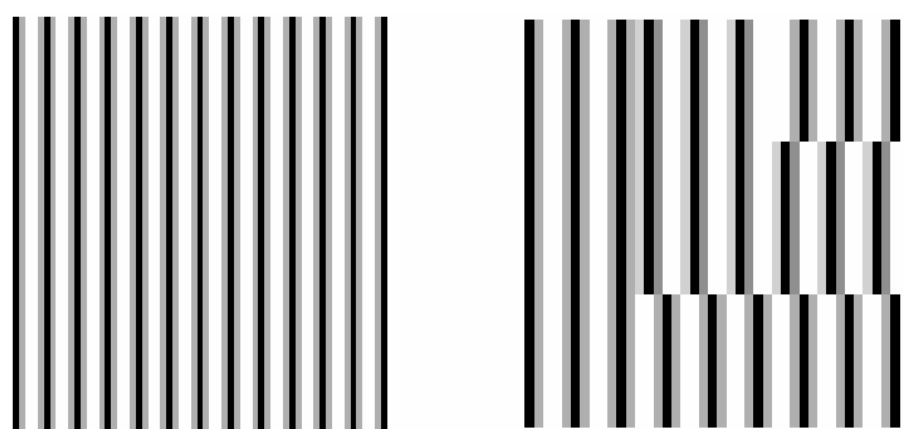

Figure-5 Fringe pattern on reference plane and on object surface
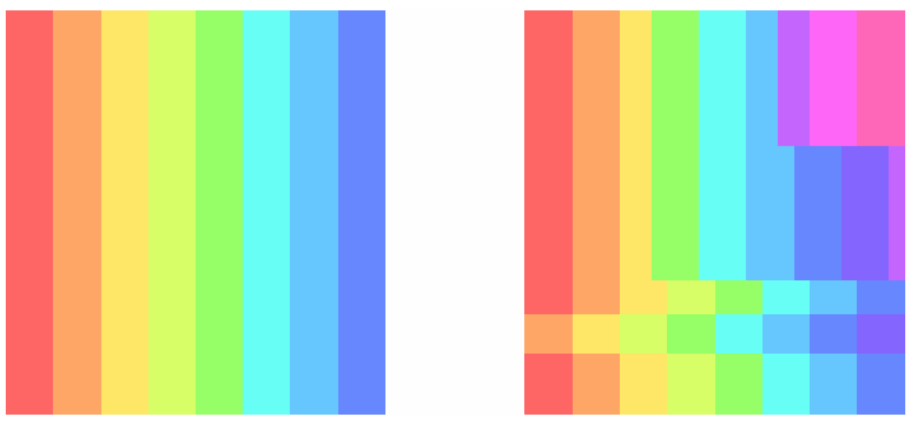

Figure- 7 (a), (b) Color-coded pattern on reference plane and on object surface

\begin{abstract}
.00.00.00 .07.07.07.14.14.14.21.21.21 .28.28.28 .49.49.49 .56.56.56 .63.63.63

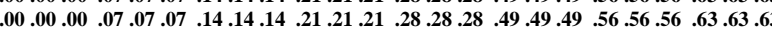

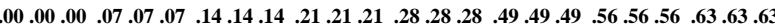
.00.00.00 .07.07.07 .14.14.14 .21.21.21 .28.28.28 .49.49.49 .56.56 .56 .63.63.63

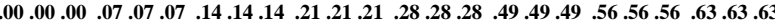

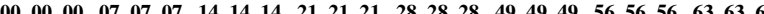
.00.00.00 .07.07.07.14.14.14.21.21. 00.00 .00 .07 .07 .07 .14 .14 .14 .21 .21 .21 .28 .28 .28 .49 .49 .49 .56 .56 .56 .63 .63 .63

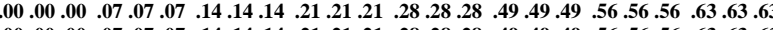
.00.00.00 .07.07.07.14.14.14 .21.21.21 .28.28.28 .49.49.49 .56 .56 .56 .63 .63 .63 .00.00.00.07.07.07.14.14.14.21.21.21.28.28.28 .49.49.49 .56.56.56 .63.63.63 .00 .00.00 .07.07.07 .14.14.14.21.21.21.28 .28.28 .49.49.49 .56 .56 .56 .63 .63 .63 .00.00.00 .07.07.07 .14.14.14.21.21.21.28 .28.28.49.49.49 .56 .56 .56 .63 .63 .63 .00.00.00 .07.07.07.14.14.14.21.21.21 .28.28.28 .49.49.49 .56.56.56 .63.63.63

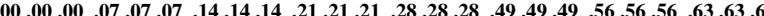
00.00 .00 .07 .07 .07 .14 .14 .14 .21 .21 .21 .28 .28 .28 .49 .49 .49 .56 .56 .56 .63 .63 .63

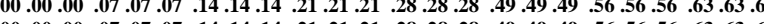

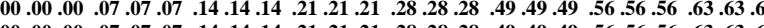
.00 .00 .00 .07 .07 .07 .14 .14 .14 .21 .21 .21 .28 .28 .28 .49 .49 .49 .56 .56 .56 .63 .63 .63

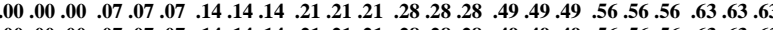
.00.00.00.07.07.07 .14.14.14.21.21.21.28 .28.28 .49.49.49 .56.56.56 .63.63.63 .00.00.00.07.07.07 .14.14.14.21.21.21.28.28.28 .49.49.49 .56.56.56 .63.63.63 .00.00.00 .07.07.07 .14.14.14 21.21.21 .28.28.28 .49.49.49 .56.56.56 .63.63.63 .00.00.00 .07.07.07 .14.14.14.21.21 .21 .28.28.28 .49.49.49 .56 .56 .56 .63.63.63 .00.00.00 .07.07.07 .14.14.14.21.21.21.28.28.28.49.49.49 .56 .56.56 .63.63.63
\end{abstract}

.00.00.00 .07.07.07.14.14.28 .21.21.49 .49.49.56 .56.70.70 .77.77.77 .91.91 .91 .00.00.00.07.07.07.14.14.28.21.21.49.49.49.56 .56.70.70.77.77.77.91.91.91 .00 .00 .00 .07 .07 .07 .14 .14 .28 .21.21.49 .49.49.56 .56.70 .70 .77.77.77 .91.91 .91 .00.00.00 .07.07.07 .14.14.28.21.21.49.49.49.56 .56 .70.70 .77.77.77 .91.91.91 .00 .00.00.07.07.07 .14.14.28 .21.21.49 .49.49.56 .56.70.70 .77.77.77 .91.91.91 .00.00.00.07.07.07.14.14.28 .21.21.49 .49.49.56 .56 .70.70 .77.77.77 .91.91 .91 .00.00.00.07.07.07 .14.14.28.21.21.49.49.49.56 .56.70.70.77.77.77.91.91.91 .00.00.00.07.07.07.14.14.28.21.21.49 .49.49.56 .56.70.70 .77.77.77 .91.91.91

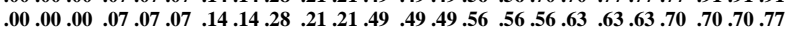
.00.00.00.07.07.07.14.14.28.21.21.49 .49.49.56 .56.56.63 .63 .63.70 .70.70.77 .00 .00.00 .07.07.07 .14.14.28 .21 .21 .49 .49.49.56 .56 .56.63 .63 .63.70 .70 .70 .77 .00 .00.00 .07.07.07 .14.14.28 .21 .21.49 .49 .49 .56 .56 .56 .63 .63 .63.70 .70 .70 .77 .00 .00.00 .07.07.07 .14.14.28 .21 .21.49 .49 .49 .56 .56 .56 .63 .63 .63 .70 .70 .70 .77 .00.00.00.07.07.07. 14.14.28.21.21.49 .49.49.56 .56.56 .63 .63 .63.70 .70.70.77 .00 .00.00 .07.07.07.14.14.28 .21 .21.49 .49.49.56 .56.56.63 .63.63.70 .70.70.77 .00.00.00.07.07.07.14.14.28.21.21.49 .49.49.56 .56.56.63 .63.63.70 .70.70.77 .00.00.00.07.07.07.14.14.14.21.21.21 .28.28.28 ..49.49.49 ..56.56.56 .63.63.63

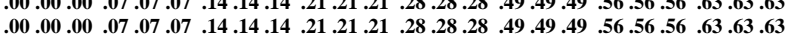

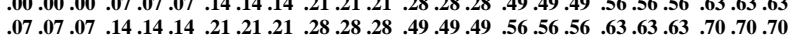

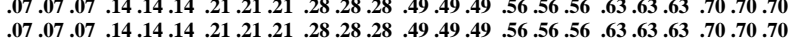
.00 .00.00 .07.07.07.14.14.14 21.21.21 .28.28.28 .49.49.49 .56.56.56 .63 .63.63 .00 .00.00.07.07.07 .14.14.14.21 .21.21 .28.28.28 .49.49.49.56.56.56 .63.63.63 .00.00 .00 .07.07.07 .14.14.14 .21.21 .21 .28.28.28 .49.49.49 .56.56.56 .63.63.63 .00 .00.00 .07.07.07 .14.14.14.21 .21.21 .28.28.28 .49.49.49.56.56.56 .63.63.63
Table 1

(a) Reference Hue Matrix (b) Object Hue Matrix 
Therefore, from the knowledge of observed color $\left(\mathrm{C}_{\mathrm{o}}\right)$ and expected color $\left(\mathrm{C}_{\mathrm{r}}\right)$, height deviation at every point on object surface can be expressed in terms of difference of their band indices $(\mathrm{m})$ as explained later. If the width of every band $(\mathrm{w})$ is made equal to the pitch of the gray-scale fringe pattern used in Takeda's method, then $\mathrm{m}$ can be directly related to $\mathrm{n}(\mathrm{x}, \mathrm{y})$ in the Eq. (6). This is the basis for determining $\mathrm{n}(\mathrm{x}, \mathrm{y})$ unambiguously.

\section{COMPUTER SIMULATIONS}

To demonstrate the ability of the proposed approach in profiling objects with surface discontinuities a typical object is simulated using MATLAB. The object simulated has three step height variations $8 \mathrm{~mm}, 11 \mathrm{~mm}$ and $32 \mathrm{~mm}$ as shown in Fig. 4. Corresponding phase modulations for the optical geometry considered are $2 \pi, 2.75 \pi$ and $8 \pi$ respectively. Fig. 5 shows the simulated fringe pattern images projected on to a reference plane and object surface respectively. Wrapped phase map $\varphi(\mathrm{x}, \mathrm{y})$ is calculated using Eq.(5) as explained in sec-2.1. Three dimensional surface plot of wrapped phase map is shown in Fig. 6. It is impossible to unwrap the phase map in Fig. 6 correctly by spatial phase unwrapping techniques, because the phase jumps at the surface discontinuities are greater than or equal to $2 \pi$. Since estimating exact number of fringes shifted at each step height is not possible from Gray-scale fringe pattern alone, we propose projection of a color-coded pattern too. Fig.7 shows images of reference plane and object respectively when color bands are projected onto them. Width of bands in the object plane is made to correspond to three pixels in image plane and eight such bands are present in the imaging window. In order to extract the necessary information from $\mathrm{C}_{\mathrm{o}}$ in determining $\mathrm{n}(\mathrm{x}, \mathrm{y})$, a procedure is established that has the following steps:

(1) Generate Reference Hue Matrix (RHM) and Object Hue Matrix (OHM) from captured images.

(2) Prepare LookUp Table (LUT) for indexing the bands.

(3) With the help of LUT generate Reference Index Matrix (RIM) and Object Index Matrix (OIM)

(4) Now calculate Difference Vector (DV), Backward Difference Matrix (BDM) and Difference Matrix (DM) from which multiples of $2 \pi$ needs to be added at each pixel can be known.

(5) Carry out phase unwrapping with the above information as shown in the flowchart.

Fig. 8 shows the flow chart of new phase unwrapping algorithm developed. All the aforementioned steps are illustrated for the simulated example considered here.

Using MATLAB RGB (Red, Green, and Blue) images can be converted to HSV (Hue, Saturation, and Value) images so that Hue of each pixel can be known. Color of each band can be identified uniquely from its corresponding Hue value. Table.1 (a), (b) shows corresponding Hue values of pixels of images in Fig.7 (a), (b) respectively. Let us refer these two matrices as Reference Hue Matrix (RHM) and Object Hue Matrix (OHM).

Next step in unwrapping procedure involves matching a detected band (Hue) with its corresponding position in the projected coded pattern. For this purpose, a Lookup Table (LUT) is generated for indexing the bands in the projected patterns as shown in Fig. 9. Using LUT, Reference Index matrix (RIM) and Object Index Matrix (OIM) are generated from RHM, OHM respectively as shown in Table-2 (a), (b). With the help of RIM and OIM one has to identify the location of surface discontinuity, determine the offset phase to be added there, and find the offset phase to be added at the beginning of every row.

In the absence of any surface discontinuity the sequence in which the color bands appear on object surface is known from $\mathrm{C}_{\mathrm{r}}$. There will be a discrepancy in the sequence of appearance of bands only if there is a surface discontinuity (See Fig.7). Therefore the presence as well as exact location of surface discontinuity can be identified with the help of a matrix (called Backward Difference matrix derived from OIM) that gives aforementioned information. Backward Difference Matrix (BD) is obtained by subtracting $\mathrm{i}^{\text {th }}$ column from $\mathrm{i}+1^{\text {th }}$ column of OIM, where $\mathrm{i}=1,2 \ldots \mathrm{N}-1$. Absolute value of any element greater than one in $\mathrm{BD}$ matrix indicates the presence of surface discontinuity at that location (that caused a phase modulation greater than $2 \pi$ in fringe pattern). The simulated object has two such discontinuities and they are well identified by looking at BD matrix shown in Table-2 (c). 


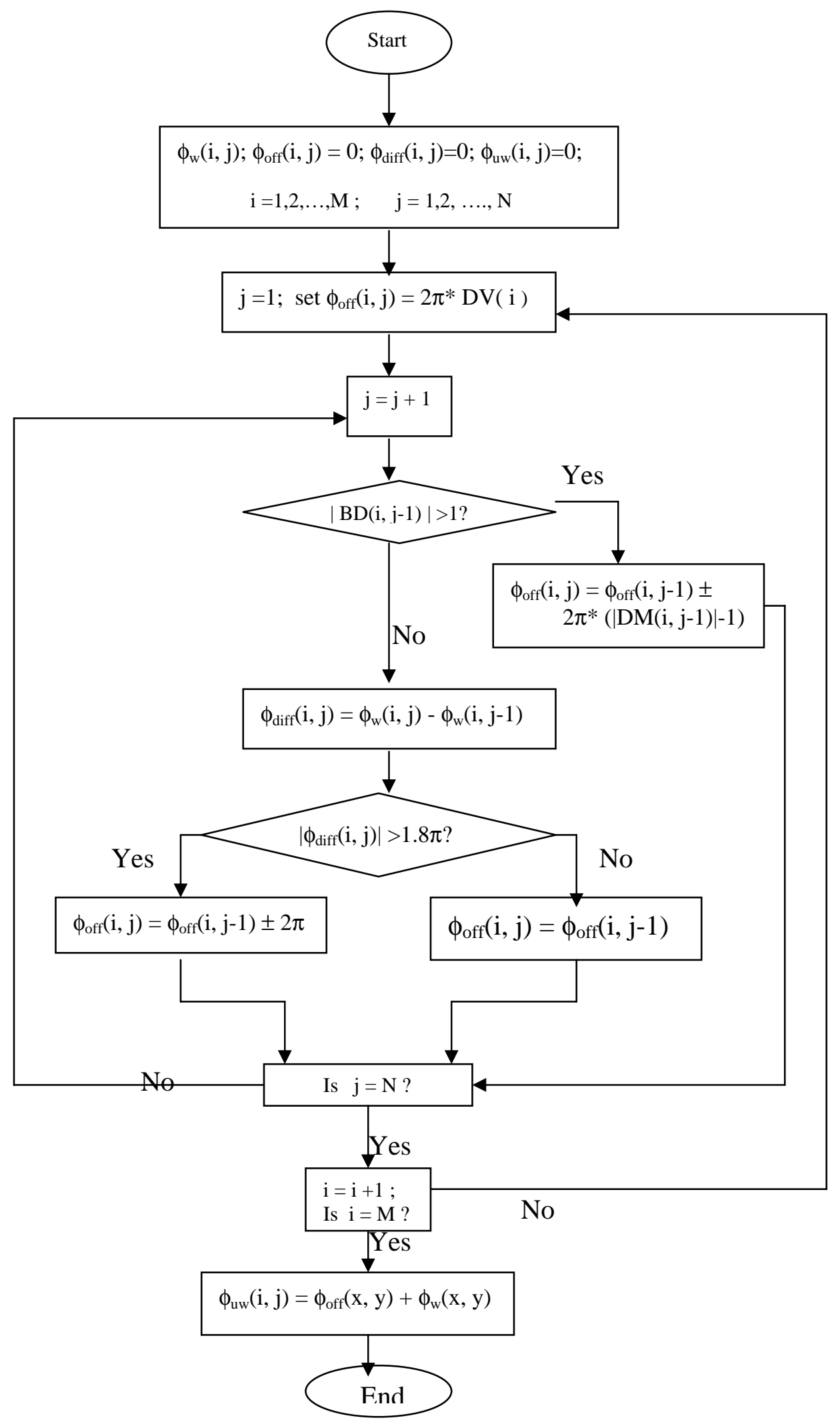

Figure-8 Flow chart for New phase unwrapping algorithm developed 


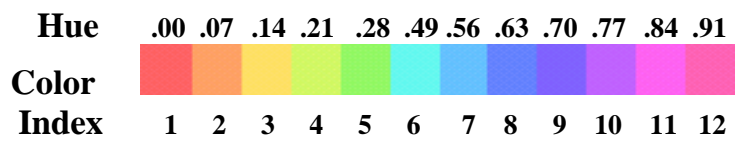

Figure-9 Look Up Table / Mapping Table Generated

Offset phase to be added at each surface discontinuity can then be calculated from the knowledge of observed color and expected color there. This information can be gained from Difference Matrix that is obtained by subtracting RIM from OIM. Offset phase to be added at the beginning of each row can be known from DV that is nothing but the first column of DM. As can be seen from Table- $2(d), 19^{\text {th }}, 20^{\text {th }}$ elements of DV are ones indicating the presence of a step height and helps in adding an offset phase of $2 \pi$ from the very beginning of corresponding rows.

Using this additional information extracted from the images captured by projecting color-coded pattern, conventional phase unwrapping procedure is modified as shown in flow chart and the resultant phase distribution is shown in Fig. 10. Fig.11 shows the comparison of wrapped phase and unwrapped phase map profiles.

111222333444555666777888 111222333444555666777888 111222333444555666777888 111222333444555666777888 111222333444555666777888 111222333444555666777888 111222333444555666777888 111222333444555666777888 111222333444555666777888 111222333444555666777888 11122233344455666777888 111222333444555666777888 111222333444555666777888 111222333444555666777888 111222333444555666777888 111222333444555666777888 111222333444555666777888 111222333444555666777888 111222333444555666777888 111222333444555666777888 111222333444555666777888 111222333444555666777888 111222333444555666777888 1112223333444555666777888 111222333444555666777888

Table 2 (a) ReferenceIndexMatrix
11122233555666771010111111121212 11122233555666771010111111121212 11122233555666771010111111121212 11122233555666771010111111121212 11122233555666771010111111121212 11122233555666771010111111121212 11122233555666771010111111121212 11122233555666771010111111121212 11122233555666677478884894910 11122233555666777888999

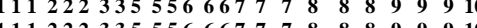
111222335556667778888899910 111222333555666777988889999910 1112223355566677778808889299910

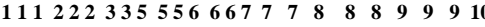

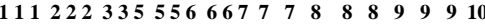

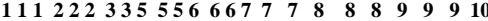
$\begin{array}{llllllllllll}111222 & 3334445555 & 6 & 6 & 6 & 7 & 7 & 7 & 8 & 8 & 8\end{array}$

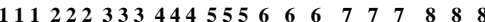
$22233344455566667477 \begin{array}{lllll}7 & 7 & 8 & 9 & 9\end{array}$ 223344455666677784899 1112522333344455567608787899 1112223334445556666777788

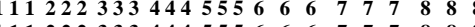

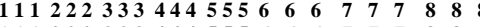

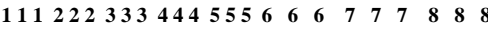

(b) Object Index Matrix
00100102001001030100100 00100102001001030100100 00100102001001030100100

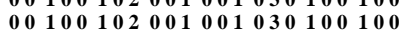
001001000

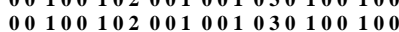

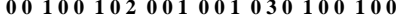
00100102001001030100100 00100102001001001001001 00100102001001001001001 0010011020001001001001001

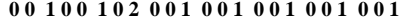
001001102000100010001001001

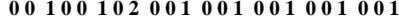

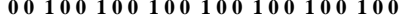
00100100100100100100100 00100100100100100100100 00100100100100100100100 00100100100100100100100

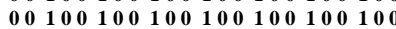

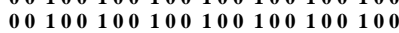

(c) Backward Difference Matrix

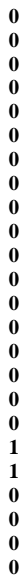

(d) DV

\begin{abstract}
000000002112112144444444 000000002112112144444444 000000002112112144444444 000000002112112144444444 000000002112112144444444 000000002112112144444444 000000002112112144444444 000000002112112144444444 000000002112112112112112 000000002112112112112112 000000002112112112112112 000000002112112112112112 000000002112112112 000000002112112112112112 00000002112112112112112 00000002112112112112112 00000000021112112112112112 000000000000000000000000000000

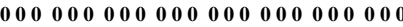
111111111111111111111111 111111111111111111111111

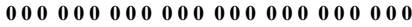

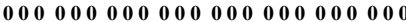

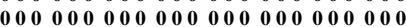

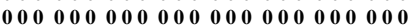

(e) Difference Matrix (DM)

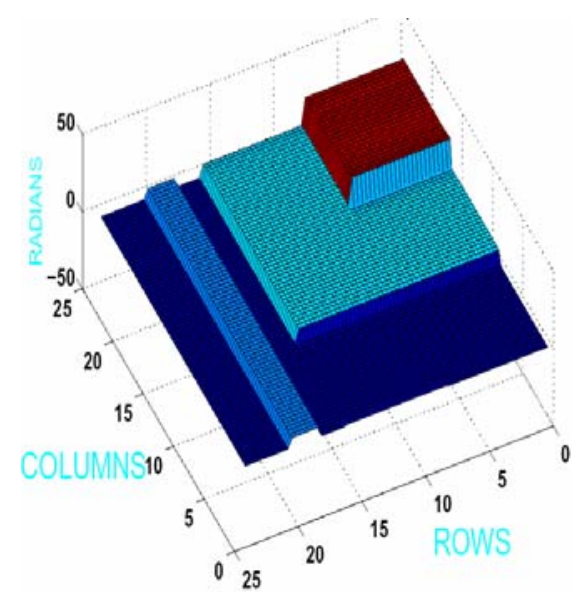

Figure-10 Result of new unwrapping algorithm

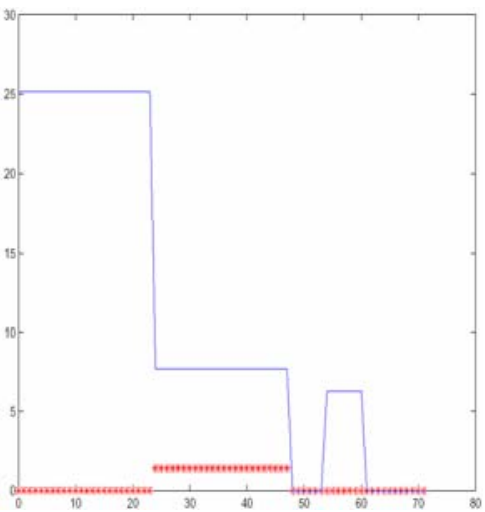

Figure-11 Comparison of wrapped and unwrapped phase values along a column. 


\section{EXPERIMENTAL RESULTS}

Gray-scale fringe pattern and color-coded patterns are generated in the PC with MATLAB 6.5. Generating these patterns using the software provides a great flexibility in setting the spatial frequency of the fringe pattern, width and color of bands to desired values. An LCD projector is used to project the generated patterns onto the surface of object under investigation. Olympus C3030 Digital CCD camera is used to record the images of the deformed fringe patterns. It automatically stores the recorded images in the Smart Media card in it. The picture data in smart media card is directly transferred to PC through USB cable with the help of CAMEDIA Master 2.0 software. Fig.12 (a), (b) shows fringe pattern on reference screen and object with two-step discontinuities respectively. Resultant image after masking the invalid data points of Fig.12 (b) is shown in Fig.12(c).

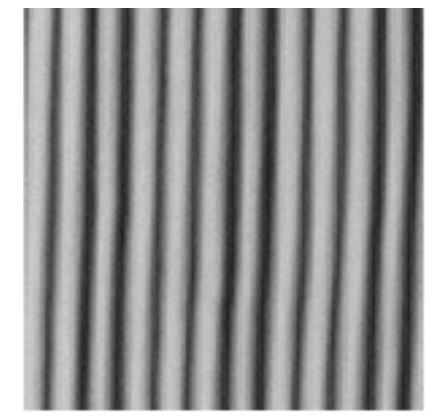

Figure.12 (a) Fringe pattern on reference plane

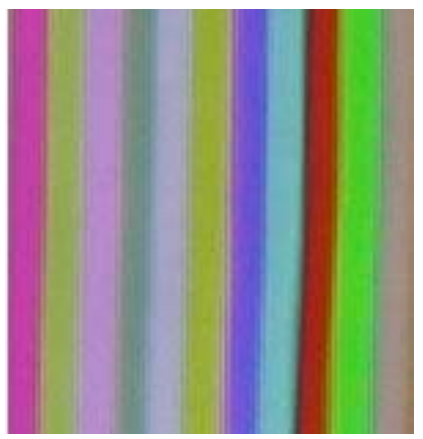

Figure.13 (a) color-coded pattern on reference plane

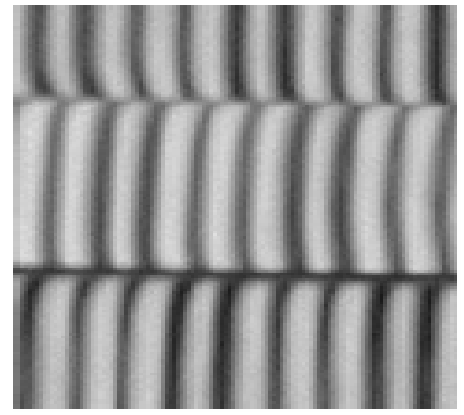

(b) on object surface

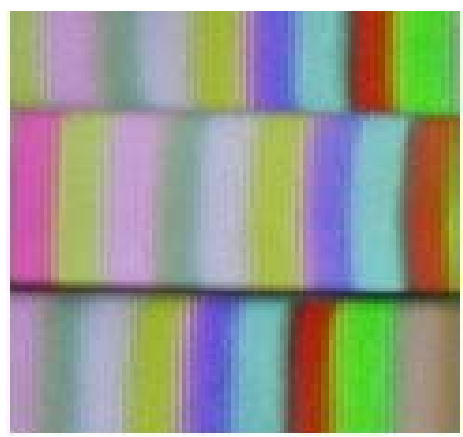

(b) on object surface

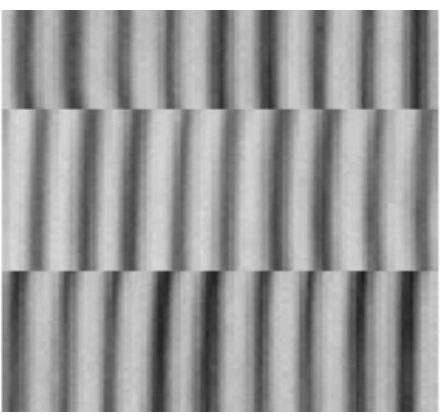

(c) resulting image after masking

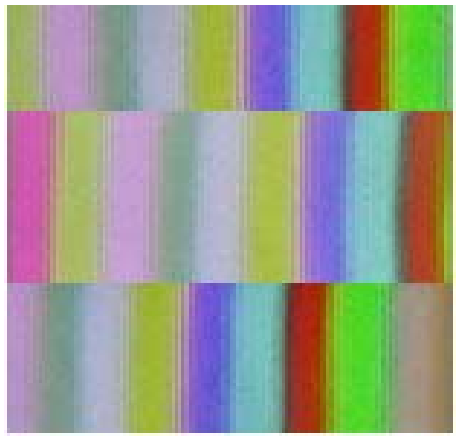

(c) resulting image after masking

Images of reference screen and object when color-coded pattern is projected on to them are shown in Figures 13 (a) and 13 (b) respectively. Fig. 14 shows the wrapped phase map of the object calculated using Takeda's method, displayed as a 3D mesh plot. It is impossible to unwrap the phase map in Fig.12(c) correctly by conventional spatial methods because the phase jumps at the steps are too large (more than $2 \pi$ ). Therefore the relative heights of the steps are uncertain to integral multiples of $2 \pi$. Even though it is impossible to determine exact numbers of fringes shifted at each step height from Gray scale fringe pattern (Fig. 12(c)), color-coded pattern on the object surface clearly reveals this information as can be seen from Fig. 13(c). All the steps described in Section-3 need to be followed for indexing the bands and finally to determine offset phase to be added to wrapped phase map to get absolute phase. Fig. 15 shows the band indices and offset phase along a column is shown in Fig.16. Three dimensional mesh plot of unwrapped phase is shown in Fig.17. 


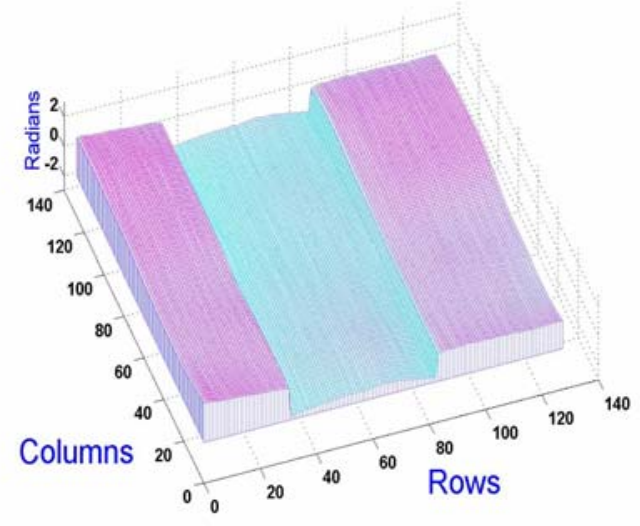

Figure. 14 Wrapped phase map calculated by Takeda's method

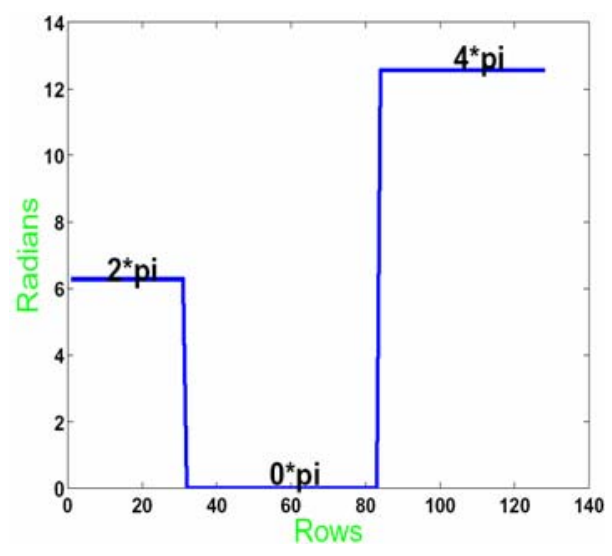

Figure.16 Offset phase to be added along each column

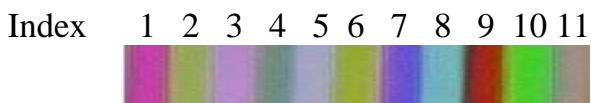

Figure. 15 Lookup table

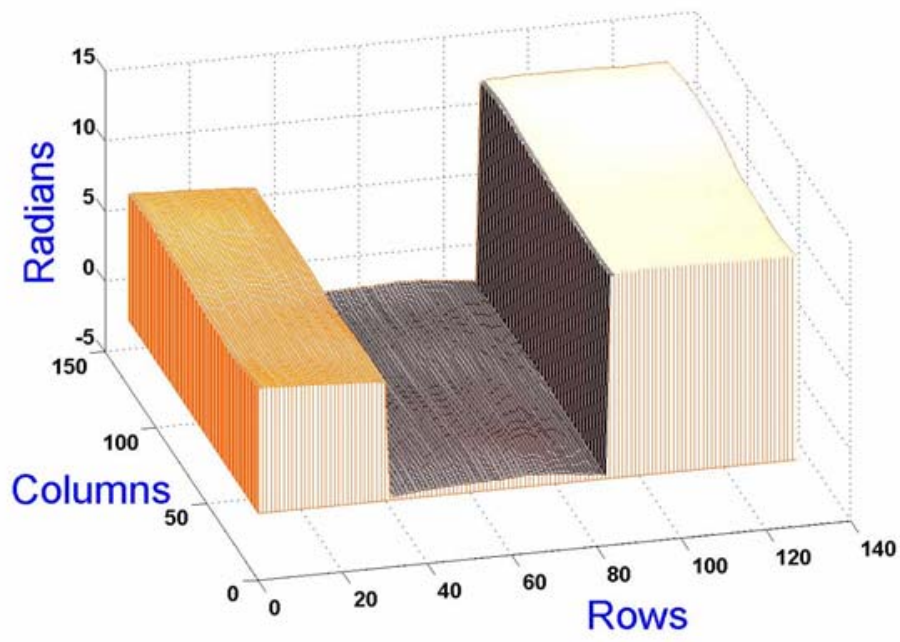

Figure.17 Three dimensional mesh plot of unwrapped phase map 


\section{DISCUSSION}

Even though color-coding strategy used in this paper assumes that each color band appears only once in the pattern, in principle any other color-coding strategy that uses few colors in coding ${ }^{12}$ can be used in conjunction with fringe projection as suggested in this paper. To be able to identify a large number of colors with out any ambiguity, there by achieve accurate results, use of high brightness LCD projectors, 3-CCD color cameras and cameras with high signal to noise ratio are recommended as pre the requirement. Since color information is used in the process, contouring results can be affected by original surface colors. For this reason meaningful results can be obtained only on surfaces with neutral color contents as is true for any of the previous technique that use color. Among several phase unwrapping techniques there exist a trade off between accuracy, fastness (no. of frames required) and reliability. Temporal phase unwrapping strategy that uses complete exponential sequence is considered to be the most reliable, accurate and fast technique as of today. (Even though the accuracy of measurement is not affected by a shorter fringe sequence used in reduced temporal phase unwrapping, a significant reduction in unwrapping reliability was reported as compared with the complete exponential sequence). New approach suggested in this paper is even superior to this method.

\section{CONCLUSIONS}

The new approached proposed, that combines color-coded projection and Fourier transform profilometry with gray scale fringe pattern projection in a specific way, has resulted in a new and more powerful method of measuring surface profile. A new procedure for exploiting the use of deformed color-coded pattern in phase unwrapping is established. Successful unwrapping of the measured phase in presence of surface discontinuities is demonstrated. Unlike temporal, spatio-temporal unwrapping and frequency multiplexing procedures, this new approach requires a single-phase map for surface profile measurement. It is shown to be significantly faster and reliable than temporal phase unwrapping that uses complete exponential sequence and compared to it the reduction both in image acquisition and in analysis times by the factor $\left[\left(\log _{2} \mathrm{~S}+1\right) / 2\right]$ is an important advantage of the present approach. Also, it has superior spatial resolution measurement capability over color-coded grating projection method.

\section{REFERENCES}

1. M. Takeda, H. Ina and S. Kobayashi, "Fourier transform method of fringe pattern analysis for computer-based topography and interferometry," J.Opt. Soc. America 72 (1), pp. 156-160, 1982.

2. V.Srinivasan, H. C. Liu and M. Halioua, "Automated phase-measuring profilometry of 3-D diffuse objects," App Opt $\mathbf{2 3}$ (18), pp. 3105-3108, 1984.

3. D.R.Burton, A. J. Goodall, J. T. Atkinson and M. J. Lalor, "The use of carrier frequency shifting for the elimination of phase discontinuities in Fourier transform profilometry," Opt Laser Eng, 23, pp. 245-257, 1995.

4. H.O. Saldner and J.M. Huntley, "Temporal phase unwrapping: application to surface profiling of discontinuous objects" App Opt 36 (13), pp. 2770-2775, 1997.

5. J.M. Huntley and H.O. Saldner, "Error-reduction methods for shape measurement by temporal phase unwrapping," J. Opt. Soc. Am. A 14 (12), pp. 3188-3195, 1997.

6. J.M. Huntley and H.O. Saldner, "Shape measurement by temporal phase unwrapping: comparison of unwrapping algorithms," Meas. Sci. Technol. 8, pp. 986-992, 1997.

7. H. Zhang, M. J. Lalor and D.R.Burton, "Spatiotemporal phase unwrapping for the measurement of discontinuous objects in dynamic fringe-projection phase-shifting profilometry," App Opt 38 (16), pp. 3534-3541, 1999.

8. H.Zhao, W. Chen and Y. Tan, "Phase unwrapping algorithm for the measurement of three dimensional object shapes," App Opt 33, pp. 4497-4500, 1994.

9. M. Takeda, Q. Gu, M. Kinoshita, H. Takai and Y. Takahashi, " Frequency-multiplex Fourier transform profilometry: a single shot three-dimensional shape measurement of objects with large height discontinuities and/or surface isolations," App Opt 36 (22), pp. 5347-5354, 1997.

10. X. Xie, M. J. Lalor, D. R. Burton and M. M. Shaw, "Phase unwrapping algorithm in the presence of discontinuities using a system with crossed grating," Opt Laser Eng 29, pp. 49-59, 1998. 
11. G. Hausler and D. Ritter, "Parallel three-dimensional sensing by color-coded triangulation," App Opt, 32. (35), pp. 7164, 1993.

12. W. Liu, Z. Wang, G. Mu and Z. Fang, "Color-coded projection grating method for shape measurement with a single exposure," App Opt 39 (20), pp. 3504-3508, 2000.

13. P. S. Huang, Q. Hu, F. Jin and F. Chiang, "Color-encoded digital fringe projection technique for high-speed three dimensional surface contouring," Opt Eng 38 (6), pp. 1065-1070, 1999.

14. L. Kinell, "Multichannel method for absolute shape measurement using projected fringes," Opt Laser Eng, 41, pp. 57-71, 2004.

15. L. Kinell and M. Sjodahl, "Robustness of reduced temporal phase unwrapping in the measurement of shape," App Opt, 40 (14), pp. 2297-2303, 2001.

16. O. A. Skydan, M. J, Lalor and D. R. Burton, "Using coloured structured light in 3-D surface measurement," Opt Laser Eng 44 (1), pp. 65-78, 2005. 